\title{
Optimality in infinite horizon discrete time models of resource management
}

\begin{abstract}
SJUR D. FLÅM†
Keywords: Optimization, resource management.

We study an infinite horizon discrete time optimization problem of the Bolza type. It is argued that this problem arises frequently in models of resource management. We obtain a characterization of optimality which is an analog to the Euler equation. The results extend those of Rockafellar and Wets (1981). Furthermore, we make no assumption about free disposal and absorbing states.
\end{abstract}

\section{Introduction}

In this paper we study the following infinite horizon problem

$$
(P) \text { minimize } L(x)=\sum_{t=1}^{\infty} L_{t}\left(x_{t-1}, \Delta x_{t}\right)
$$

over all bounded sequences $x=\left(x_{t}\right)_{t=0}^{\infty}$ in $R^{n}$.

Here $\Delta x_{t}=x_{t}-x_{t-1}$ and $L_{t}: R^{n} \rightarrow(-\infty, \infty], t=1,2, \ldots$ are lower semicontinuous convex functions, none of which is identically $+\infty$. We assume that for every bounded sequence $\left(x_{t}\right)_{t=0}^{\infty}$ in $R^{n},\left(L_{t}\left(x_{t-1}, \Delta x_{t}\right)\right)_{t=1}^{\infty}$ majorizes at least one absolutely convergent sequence in $R^{1}$. For this reason we adopt the convention that $L(x)=+\infty$ when the series $\sum_{t=1}^{\infty} L_{t}\left(x_{t-1}, \Delta x_{t}\right)$ does not converge. In order to exclude pathological cases we will also assume that inf $L(x)>-\infty$.

We emphasize that $L$ and $L_{t}, t=1,2, \ldots$ are convex functions into the extended real line. The cost $+\infty$ represents the fact that certain trajectories are impossible or forbidden.

Specifically, $L_{t}\left(x_{t-1}, \Delta x_{t}\right)=+\infty$ means that the pair $x_{t-1}, x_{t}$ violates some implicit constraints. For example, $L_{t}$ may be defined by

$$
L_{t}\left(x_{t-1}, \Delta x_{t}\right)= \begin{cases}+\infty & \text { if }\left(x_{t-1}, x_{t}\right) \notin C_{t} \\ l_{t}\left(x_{t-1}, \Delta x_{t}\right) & \text { otherwise }\end{cases}
$$

If $C_{t}$ is a convex set and $l_{t}$ is a convex function then $L_{t}$ is a convex function. The fisheries model in $\S 2$ will make this point more clear.

Note that $(P)$ resembles problems of Bolza type in the classical calculus of variations, the difference being that time is now discrete and the horizon is infinite. Recall that perturbations and partial integration play a vital role in the derivation of the Euler equation. Therefore it should come as no big surprise that in the discrete case perturbations and partial summation come to the front stage.

Received 3 August 1983.

† The Chr. Michelsen Institute, N-5036 Fantoft, Norway. 
The paper is outlined as follows. Section 2 describes the importance of the problem in resource management. For concreteness, we illustrate by means of a standard model of fisheries management. Section 3 deals with a strong characterization of optimality in terms of dual variables. In $\S 4$ we demonstrate that the latter variables arise as a solution to a dual optimization problem. These results have all been obtained in the finite horizon case by Rockafellar and Wets (1981). The final section provides interpretation of the results.

\section{A fisheries model}

The importance of $(P)$ in resource management, where a long run dynamic perspective is required, cannot be overstated. As an example consider the following. Let $c_{t-1} \in R_{+}{ }^{n}$ be the catch of fish and let $x_{t-1} \in R_{+}{ }^{n}$ be the escapement after catch at the beginning of period $t-1$. Here $R_{+}{ }^{n}$ denotes the set of all $n$-vectors with nonnegative coordinates. The dynamics are governed by the equation

$$
x_{t}=f_{t-1}\left(x_{t-1}\right)-c_{t}, \quad t \geqslant 1
$$

and the profit in period $t$ is $\pi_{t}\left(x_{t-1}, c_{t}\right)$.

Let $\delta, 0<\delta<1$ be a discount fact and let the initial point $\bar{x}_{0} \geqslant 0$ be given. The objective is to maximize the present value

$$
\sum_{t=1}^{\infty} \delta^{t} \pi_{t}\left(x_{t-1}, c_{t}\right)
$$

We translate this optimization problem into the form $(P)$ by defining

$$
L_{1}\left(x_{0}, \Delta x_{1}\right)= \begin{cases}-\delta \pi_{1}\left(x_{0}, f_{0}\left(x_{0}\right)-x_{1}\right) & \text { if } x_{0}=\bar{x}_{0}, x_{1} \geqslant 0 \text { and } x_{1} \leqslant f_{0}\left(x_{0}\right) \\ +\infty & \text { otherwise }\end{cases}
$$

and for $t>1$

$$
L_{t}\left(x_{t-1}, \Delta x_{t}\right)= \begin{cases}-\delta^{t} \pi_{t}\left(x_{t-1}, f_{t-1}\left(x_{t-1}\right)-x_{t}\right) & \text { if } x_{t-1}, x_{t} \geqslant 0, x_{t} \leqslant f_{t-1}\left(x_{t-1}\right) \\ +\infty & \text { otherwise }\end{cases}
$$

Assume that $\pi_{t}$ and $f_{t-1}$ are both concave and continuous for all $t \geqslant 1$. Also suppose that $\pi_{t}\left(x_{t-1}, c_{t}\right)$ is monotonically non-decreasing in $c_{t}$. Then it is straightforward to demonstrate that $L_{t}$ is convex and lower semi-continuous.

\section{Characterization of optimality}

The purpose of this section is to demonstrate strong (necessary and sufficient) duality relations. After defining some notations we give in Theorem 1 a sufficient condition for optimality in terms of dual variables. For a necessary condition an assumption about strict feasibility will be made (a 'Slater' condition). When this condition is in force we are able to demonstrate (Theorem 2 ) that dual variables exist in a neat form.

For notational convenience denote by $l_{n}^{\infty}$ the set of bounded sequences in $R^{n}$, and let $l_{n}^{1}$ be the set of sequences $\left(p_{t}\right)_{t=1}^{\infty}$ in $R^{n}$ such that $\sum_{t=1}^{\infty}\left|p_{t}\right|<\infty$. 
Both these spaces are so-called Lebesgue spaces. They are in fact Banach spaces and $l_{n}^{\infty}$ is the dual of $l_{n}{ }^{1}$ under the natural pairing

$$
\sum_{t=1}^{\infty} p_{t} \cdot y_{t}
$$

Now define

$$
\phi: l_{n}^{\infty} \rightarrow[-\infty, \infty] \text { by } \phi(y)=\inf _{x \in l_{n}^{\infty}} \sum_{t=1}^{\infty} L_{t}\left(x_{t-1}, \Delta x_{t}+y_{t}\right)
$$

Clearly $\phi(y)$ is convex. Observe that $y \in l_{n}^{\infty}$ plays the role of a perturbation. In general denote by $\partial g(z)$ the set of subgradients of $g$ at $z$.

\section{Theorem 1}

Let $x \in l_{n}{ }^{\infty}$ and $p \in l_{n}{ }^{1}$. Then $x$ is an optimal solution of $(P)$ and $p \in \partial \phi(0)$ if and only if $\left(\Delta p_{t}, p_{t}\right) \in \partial L_{t}\left(x_{t-1}, \Delta x_{t}\right)$ for all $t \geqslant 1$ where $\Delta p_{t}=p_{t}-p_{t-1}$.

\section{Proof}

$p \in \partial \phi(0)$ means that $\phi(0) \leqslant \phi(y)-p y$ for all $y \in l_{n}^{\infty}$. Consequently $x$ solves $(P)$ and $p \in \partial \phi(0)$ if and only if

$$
\sum_{t=1}^{\infty} L_{t}\left(x_{t-1}^{\prime}, \Delta x_{t}^{\prime}+y_{t}\right)-\sum_{t=1}^{\infty} p_{t} \cdot y_{t}
$$

attains its minimum at $x^{\prime}=x$ and $y=0$.

Now define $z_{t}=x_{t-1}^{\prime}$ and $w_{t}=\Delta x_{t}^{\prime}+y_{t}$ for $t=1,2, \ldots$ Let $p_{0}=0$. Then

$$
\begin{aligned}
\sum_{t=1}^{T} p_{t} \cdot y_{t} & =\sum_{t=1}^{T} p_{t} \cdot\left(w_{t}-\Delta x_{t}^{\prime}\right) \\
& =\sum_{t=1}^{T} p_{t} \cdot w_{t}-p_{T} \cdot x_{T}^{\prime}+\sum_{t=1}^{T} \Delta p_{t} \cdot x_{t-1}^{\prime}
\end{aligned}
$$

Here $p_{T} \cdot x^{\prime}{ }_{T} \rightarrow 0$ when $T \rightarrow \infty$. Hence after this change of variables (1) becomes

$$
\sum_{t=1}^{\infty}\left\{L_{t}\left(z_{t}, w_{t}\right)-\Delta p_{t} \cdot z_{t}-p_{t} \cdot w_{t}\right\}
$$

We are to minimize (2) over all $z, w \in l_{n}^{\infty}$. Note that the criterion (2) is separable.

Furthermore, $L_{t}\left(z_{t}, w_{t}\right)-\Delta p_{t} \cdot z_{t}-p_{t} \cdot w_{t}$ attains its minimum at $\left(x_{t-1}, \Delta x_{t}\right)$ if and only if $\left(\Delta p_{t}, p_{t}\right) \in \partial L_{t}\left(x_{t-1}, \Delta x_{t}\right)$. This completes the proof. Q.E.D.

\section{Remark 1}

The significance of this result is that for purposes of computation or planning, the dual variables allow for the optimization problem $(P)$ to be completely decomposed into an infinite sequence of independent subproblems. In its original version any perturbation of $x_{t-1}$ affects not only $L_{t}\left(x_{t-1}, \Delta x_{t}\right)$ but also $L_{s}\left(x_{s-1}, \Delta x_{s}\right)$ for all $s>t$. By contrast, suppose $L_{t}$ is strictly convex. Then the choice of $z_{t}, w_{t}$ so as to minimize $L_{t}\left(z_{t}, w_{t}\right)-\Delta p_{t} \cdot z_{t}-p_{t} \cdot w_{t}$ is completely free from all $t \geqslant 1$ in the sense that it has no future effects. In general, when $L_{t}\left(z_{t}, w_{t}\right)-\Delta p_{t} \cdot z_{t}-p_{t} \cdot w_{t}$ does not have a unique minimum $\left(z_{t}, w_{t}\right)$ we must check the feasibility requirement: $z_{t}+w_{t}=z_{t+1}$. 
Remark 2

The dual variables $p=\left(p_{t}\right)_{t=1}^{\infty}$ may be interpreted as follows. Fix a price $p_{t}$ for perturbing the rate of change in period $t$. Whenever a perturbation profile $y=$ $\left(y_{t}\right)_{t=1}^{\infty}$ is chosen we incur an additional cost $p y=\sum_{t=1}^{\infty} p_{t} \cdot y_{t}$.

It follows that for a given price regime $p$ on perturbations $\phi(y)-p y$ is the lowest possible cost. We say that $p$ is an 'equilibrium price' regime if no perturbation pays off. This means that $\phi(0) \leqslant \phi(y)-p(y)$, i.e. $p \in \partial \phi(0)$. Theorem 1 says that minimum cost is achieved at $x$ and $p$ is an equilibrium price regime if $p$ supports the optimal trajectory in the sense that $\left(\Delta p_{t}, p_{t}\right) \in \partial L_{t}\left(x_{t-1}, \Delta x_{t}\right)$ for all $t \geqslant 1$.

\section{Remark 3}

Theorem 1 provides a sufficient condition for optimality, i.e. if $x \in l_{n}^{\infty}, L(x)<\infty$, $p \in l_{n}{ }^{1}$ and $\left(\Delta p_{t}, p_{t}\right) \in \partial L_{t}\left(x_{t-1}, \Delta x_{t}\right)$, for all $t \geqslant 1$, then $x$ solves $(P)$. We would also want a necessary characterization of optimality saying that if $x \in l_{n}^{\infty}$ is optimal in $(P)$ then there exists $p \in l_{n}{ }^{1}$ such that $\left(\Delta p_{t}, p_{t}\right) \in \partial L_{t}\left(x_{t-1}, \Delta x_{t}\right)$. For this purpose two additional questions must be addressed. First, remember that $p \in \partial \phi(0)$. Hence we must guarantee that $\partial \phi(0)$ is non-empty. Second, since $\phi$ is a convex functional from $l_{n}^{\infty}$ to $[-\infty, \infty]$ we have $\partial \phi(y) \subseteq\left(l_{n}^{\infty}\right)^{*}$ at any $y \in l_{n}^{\infty}$. However, $l_{n}{ }^{1}$ is not reflexive, i.e. $\left(l_{n}{ }^{1}\right)^{* *}=$ $\left(l_{n}^{\infty}\right)^{*} \supseteq l_{n}{ }^{1}$ where the last inclusion is strict (Brown and Pearcy 1979). In order to have an amenable representation of $p \in \partial \phi(0)$ we want $\partial \phi(0)$ to be a subset of $l_{n}{ }^{1}$. Both these questions are resolved in the following theorem.

\section{Theorem 2}

Suppose there exists $\epsilon>0$, suppose $x \in l_{n}^{\infty}$ is optimal and a summable sequence $\left(\alpha_{t}\right)_{t=1}^{\infty}$ of non-negative real numbers such that for all $t \geqslant 1 L_{t}(z, w) \leqslant \alpha_{t}$ when $\left|z-x_{t-1}\right| \leqslant \epsilon,\left|w-\Delta x_{t}\right| \leqslant \epsilon$. Then $\partial \phi(0)$ is non-empty. Furthermore, $\partial \phi(0)$ may be identified with a weakly compact subset of $l_{n}{ }^{1}$. It follows that for every optimal solution $x$ of $(P)$ a $p \in l_{n}{ }^{1}$ exists such that $\left(\Delta p_{t}, p_{t}\right) \in \partial L_{t}\left(x_{t-1}, \Delta x_{t}\right)$ for all $t \geqslant 1$.

\section{Proof}

Let $y_{t} \in R^{n}$ be such that $\left|y_{t}\right| \leqslant \epsilon$ for every $t \geqslant 0$. Define $x_{t}^{\prime}=x_{t}-y_{t}$ for $t \geqslant 0$ and $z_{t}=x_{t-1}^{\prime}, w_{t}=\Delta x_{t}^{\prime}+y_{t}$ for $t \geqslant 1$. Then $z_{t}-x_{t-1}=-y_{t-1}$ and $w_{t}-\Delta x_{t}=y_{t-1}$. Consequently

$$
\sum_{t=1}^{\infty} L_{t}\left(x_{t-1}^{\prime}, \Delta x_{t}^{\prime}+y_{t}\right)=\sum_{t=1}^{\infty} L_{t}\left(z_{t}, w_{t}\right) \leqslant \sum_{t=1}^{\infty} \alpha_{t}
$$

Hence $\phi(y)<\infty$ for all $y \epsilon l_{n}^{\infty}$ with $|y| \leqslant \epsilon$. It follows that $0 \in$ int $\{y \mid \phi(y)<\infty\}$ and this suffices to guarantee that $\partial \phi(0)$ is non-empty. The next statement of the theorem follows from corollary 2c of Rockafellar (1971). The final assertion is part of Theorem 1. This completes the proof. Q.E.D.

\section{Duality}

In this section we show that the price profile $p \in l_{n}{ }^{1}$ supporting an optimal trajectory may arise as the solution to a dual problem. This latter problem may be stated as

$$
\text { (D) maximize } \sum_{t=1}^{\infty} L_{t}\left(\Delta p_{t}, p_{t}\right) \text { over all } p \in l_{n}{ }^{1}
$$


Here $L_{t}: R^{n} \times R^{n} \rightarrow[-\infty, \infty)$ is defined by

$$
\boldsymbol{L}_{t}(\alpha, \beta)=\inf _{a, b \in R^{n}}\left\{\left(L_{t}(a, b)-\alpha \cdot a-\beta \cdot b\right\}\right.
$$

Let inf $(P)$, sup $(D)$ denote the optimal value of $(P)$ and $(D)$ respectively.

\section{Theorem 3}

We always have inf $(P) \geqslant \sup (D)$. Furthermore $p \in \partial \phi(0) \cap l_{n}{ }^{1}$ if and only if $\inf (P)=$ $\max (D)$ and $p$ is optimal for $(D)$.

\section{Proof}

Clearly

$$
\begin{aligned}
\inf (P) & =\phi(0) \geqslant \inf _{y \in I_{t}^{\infty}}\{\phi(y)-p y\}=\inf _{x, y \in I_{n}^{\infty}} \sum_{t=1}^{\infty} L_{t}\left(x_{t-1}, \Delta x_{t}+y_{t}\right)-p y \\
& =\inf _{z, w \in I_{n}^{\infty}} \sum_{t=1}^{\infty}\left\{L_{t}\left(z_{t}, w_{t}\right)-\Delta p_{t} \cdot z_{t}-p_{t} w_{t}\right\} \geqslant \sum_{t=1}^{\infty} L_{t}\left(\Delta p_{t}, P_{t}\right)
\end{aligned}
$$

Here we applied the same change of variable as in the proof of Theorem 1. It follows that inf $(P) \geqslant \sup (D)$ and equality holds if and only if $\phi(0)+p y \leqslant \phi(y)$ for all $y \in l_{n}^{\infty}$, i.e. $p \in \partial \phi(0)$. Q.E.D.

\section{Interpretation of results}

We want to conclude by restating some of the preceding results in the context of the fisheries model. It goes without saying that the same results apply to models of capital growth. Let $P_{t}=\delta^{-t} p_{t}$. In this case the condition $\left(\Delta p_{t}, p_{t}\right) \in \partial L_{t}\left(x_{t-1}, \Delta x_{t}\right)$ means that

$$
\delta \pi_{t}\left(x_{t-1}^{\prime}, c_{t}^{\prime}\right)+\left(\delta P_{t} x_{t}^{\prime}-P_{t-1} x_{t-1}^{\prime}\right) \leqslant \delta \pi_{t}\left(x_{t-1}, c_{t}\right)+\left(\delta P_{t} x_{t}-P_{t-1} x_{t-1}\right)
$$

for all $x_{t-1}^{\prime}, c^{\prime}{ }_{t}$ with $x_{t}^{\prime}=f_{t}\left(x_{t-1}^{\prime}\right)-c_{t}^{\prime}$ and $x_{t}=f\left(x_{t-1}\right)-c_{t}$. This says of course that the sum of current profit and the capital gain, the latter being evaluated at prices $\left(P_{t}\right)_{t=1}^{\infty}$, is maximal along the optimal trajectory. These prices might be termed a regime of 'equilibrium' (or efficiency) prices. This word underlines the salient feature that under these prices even a short-sighted manager would not find it profitable to deviate from the long-run optimal trajectory. In fact, Theorem 1 says that if an equilibrium price regime exists (and $\pi_{t}$ is strictly concave) then we may perform the dynamic optimization by simply maximizing the current sum of profit and capital gain in a completely myopic manner. Conversely, suppose that for some $\epsilon>0$ and $x, c \in l_{n}^{\infty}$, that $\pi_{t}\left(x_{t}^{\prime}, c_{t}^{\prime}\right) \geqslant \beta_{t}$ when $\left|x_{t}^{\prime}-x_{t}\right| \leqslant \epsilon$ and $\left|c^{\prime}{ }_{t}-c_{t}\right| \leqslant \epsilon$ where $\sum_{t=1}^{\infty} \delta^{t}\left|\beta_{t}\right|<\infty$. Then by Theorem 2, if $x \in l_{n}^{\infty}$ is optimal there exists an equilibrium price regime $P$ supporting $x$.

\section{Conclusions}

We have demonstrated that optimality in some convex infinite horizon models is characterized in terms of an equilibrium price system. These prices support the optimal trajectory in such a way that the mathematical programming problem is decomposed with respect to time. Similar results have been obtained by several 
authors. See Weitzman (1973), Polterovich (1983), McKenzie (1976). However, it is common practice in these papers to invoke assumptions about free disposal and 'nothing ventured nothing gained' or to assume some kind of compactness. In our proof we have completely avoided such restrictions. Also our proof does not depend on any argument from the realm of dynamic programming.

We emphasize that the characterization

$$
\left(\Delta p_{t}, p_{t}\right) \in \partial L_{t}\left(x_{t-1}, \Delta x_{t}\right), t \geq 1
$$

is a discrete time version of the well-known Euler equation

$$
\frac{d}{d t} \frac{\partial}{\partial \dot{x}} L_{t}(x(t), \dot{x}(t))=\frac{\partial}{\partial x} L_{t}(x(t), \dot{x}(t))
$$

To see this, define

$$
p(t)=\frac{\partial}{\partial \dot{x}} L_{t}(x(t), \dot{x}(t))
$$

Then by (4) and (5) we get

$$
(\dot{p}(t), p(t))=\nabla L_{t}(x(t), \dot{x}(t))
$$

In the non-smooth case (6) becomes

$$
(\dot{p}(t), p(t)) \in \partial L_{t}(x(t), \dot{x}(t))
$$

Now discretize (7) to get (3). We conclude by showing that in economic applications convexity is often very appropriate but differentiability assumptions are rather unnatural. To this end we elaborate slightly on the fisheries example of $\S 2$. Let $y_{t}$ be the activity vector of the fishing industry in period $t$. Suppose $y_{t}$ is chosen so as to maximize the profit $a_{t} \cdot y_{t}$ subject to the constraints

$$
A_{t} Y_{t} \leqslant b_{t}\left(x_{t}, x_{t+1}\right), Y_{t} \geq 0
$$

Suppose also that each coordinate of $b_{t}$ is a concave function of $x_{t}, x_{t+1}$. Then it is easy to show that the optimal value $\pi_{t}\left(x_{t}, x_{t+1}\right)$ is indeed a concave function of $x_{t}, x_{t+1}$. However, we can not expect $\pi_{t}$, being the optimal value of a $L P$-problem, to be differentiable with respect to the data in a classical sense. This explains why the relaxation to subdifferentiability is appropriate and not only of academic interest.

\section{REFERENCES}

BazaraA, M. S., and Sherty, C. M. (1979). Non-linear Programming; Theory and Algorithms (John Wiley: New York).

Brown, A., and PeARCY, C. (1979). Introduction to operator theory, I (Springer Verlag: New York).

KAMIEN, M. I., and SchWARTZ, N. L. (1981). Dynamic optimization; The calculus of variations and optimal control in economics and management (North Holland, New York).

Rockafellak, R. T. (1971). Integrals which are convex functionals, II. Pacific Journal of Mathematics, 39, 439-468.

Rockafellar, R. T., and Wets, R. J.-B. (1981). Deterministic and stochastic optimization problems of Bolza type in discrete time. Working Paper 81-69, IIASA.

MCKENZIE, L. W. (1976). Turnpike theory. Econometrica, 44, 841-865.

Polterovich, W. M. (1983). Equilibrium trajectories for economic growth. Econometrica, 51, 693-723.

Weitzman, M. L. (1973). Duality theory for infinite horizon convex model, Management Science, 19, 783-789. 